- Case Report

\title{
Acquired Hemophilia in a Patient Presenting with Swollen Left Limb
}

\author{
George Wong Pah Kiam* \\ School of Medical Science, Health Campus, Universiti Sains Malaysia, Kubang Kerian, Malaysia
}

Acquired hemophilia is a rare, potentially life-threatening disease that usually remains under-recognized especially in a primary setting; thus, diagnosing this disease is very challenging. Given its prevalence in elderly patients, awareness and diagnosis of this condition in the aging population (particularly those with unexplained bleeding or prolonged activated partial thromboplastin time) should be improved and better managed by the clinicians. Early diagnosis and prompt treatment are usually effective in preventing the adverse outcomes of this disease. In this report, we discuss a case of an elderly patient with acquired hemophilia who initially presented with swelling of his lower left limb. The diagnosis of acquired hemophilia was made a month after the appearance of symptoms. Early diagnosis with proper treatment could have been provided to this patient, if the initial assessment had been thoroughly conducted.

Keywords: Acquired Hemophilia; Aged; Mixing Test; Factor VIII

Received: November 8, 2017, Revised: February 1, 2018, Accepted: February 2, 2018

*Corresponding Author: George Wong Pah Kiam https://orcid.org/0000-0003-3351-2523

Tel: +60-0128841680, Fax: +60-09-7653370, E-mail: geominiz@yahoo.com 


\section{INTRODUCTION}

Acquired hemophilia is an autoimmune disease characterized by the production of autoantibodies against plasma coagulation factors. ${ }^{1)}$ It is a rare but potentially life-threatening condition that occurs more commonly in the elderly population. ${ }^{2)}$ The diagnosis of this condition is under-recognized, and a delay in diagnosis leads to a high mortality rate among these patients. In the elderly group of patients, the unexplained bleeding tendency with isolated prolonged activated partial thromboplastin time (aPTT) should raise high clinical suspicion of acquired hemophilia. Treatment of acquired hemophilia includes acute bleeding control and suppression of the autoimmune process by an immunosuppressive agent. ${ }^{3,4)}$

\section{CASE REPORT}

A 67-year-old man with underlying ischemic heart disease and dyslipidemia presented to the emergency department with spontaneous left leg swelling for a week. There were no complaints of any calf pain or bleeding tendency elsewhere. The patient had no history of preceding trauma or consumption of traditional supplements. Clinical examination revealed a hematoma measuring $10 \mathrm{~cm} \times 6 \mathrm{~cm}$ in the left calf; however, there was no evidence of hepatosplenomegaly. No other signs of bleeding were noted. The patient did not report of any personal or family history of bleeding disorders. He was compliant with his medications, which included Tab Ramipril (5 mg daily) and Tab Atorvastatin (40 mg, taken at night).

He was initially suspected to have lower left limb deep vein thrombosis; however, the results of D-dimer and ultrasound Doppler tests of the affected limb were found to be negative. The prothrombin ratio and platelet counts were normal but the aPTT was prolonged (55.8 seconds) relative to control (30-45 seconds). Following his recovery, he was discharged from casualty and referred to primary care for a follow-up examination of the swelling.

During subsequent visits to the clinic, the patient was attended by

Table 1. Patient's laboratory data

\begin{tabular}{lcc}
\hline \multicolumn{1}{c}{ Blood investigation } & Result & Normal range \\
\hline Full blood count & & \\
$\quad$ Hemoglobin $(\mathrm{g} / \mathrm{dL})$ & 11.5 & $13.5-17.4$ \\
Red blood cell $\left(\times 10^{6}\right)$ & 4.29 & $4.2-6.1$ \\
White blood cell $\left(\times 10^{9}\right)$ & 8.09 & $3.8-9.7$ \\
Hematocrit $(\%)$ & 36.4 & $37.5-49.8$ \\
Platelet $\left(\times 10^{3}\right)$ & 264 & $167-176$ \\
Prothrombin time (s) & 14.3 & $12.6-15.7$ \\
Activated partial thromboplastin time (s) & 59.7 & $30.0-45.8$ \\
Factor VIII levels (\%) & 5 & $50-190$ \\
Factor IX (\%) & 124 & $57-160$ \\
Factor XI (\%) & 68.7 & \\
Factor VIII inhibitor $(\%)$ & Detected \\
Thrombin time $(\mathrm{s})$ & 16.2 & \\
Lupus anticoagulant & Negative & \\
\hline
\end{tabular}

different doctors; however, his condition remained the same with prolonged isolated aPTT ranging between 51-59 seconds. Several years ago, the patient had been tested for anemia and presented a normal coagulation profile at the time. In the absence of extensive hematoma and other bruises, no further investigation was carried out for the abnormally prolonged aPTT.

During the 4th week of the illness, he developed new bruises on both of his hands. There was bleeding from venepuncture site, which did not stop spontaneously. However, there were no changes in the leg hematoma, and the repeated aPTT values still remained high.

On family medicine specialist review, he was referred to hematological team based on clinical suspicion of acquired hemophilia. Upon admission, the patient was actively bleeding from the venepuncture site. Mixing studies with the patient's plasma were performed but the prolonged aPTT failed to correct completely. Further, the patient had a low plasma factor VIII level of 5\%. The plasma VIII inhibitors were also detected, and the titre value was found to be $19.6 \mathrm{BU} / \mathrm{mL}$. Lupus anticoagulant was not detected in patient's plasma (Tables 1,2).

Following the diagnosis of acquired hemophilia, the patient was subjected to immunosuppressive therapy in the form of high dose prednisolone $1 \mathrm{mg} / \mathrm{kg}$ per day (total $70 \mathrm{mg}$ daily) and cyclophosphamide (100 mg once a day). He was later treated with rituximab (375 $\mathrm{mg} / \mathrm{m}^{2}$ on a weekly basis) for a 4 -week duration. The patient was also given a short period of inpatient treatment for rehabilitation and rest, allowing the bleeding to stop and hematoma to be resolved. Finally, he was discharged when his aPTT was corrected to normal range.

\section{DISCUSSION}

Acquired hemophilia is a rare spontaneous autoimmune disorder caused by the development of antibodies against its own plasma coagulation factors, most commonly factor VIII. ${ }^{1,3)}$ Acquired hemophilia patients are distributed worldwide, with the incidence of 1.48 per million persons per year. ${ }^{5)}$ It typically affects the middle-aged group (6080 years old) and is reported to occur at equal rates in both sexes. ${ }^{1)} \mathrm{Nu}-$ merous conditions can give rise to this disorder; however, approximately $50 \%$ cases are idiopathic. ${ }^{1)}$ More severe forms of hemophilia have been observed predominantly in male patients. ${ }^{3)}$

Diagnosis of acquired hemophilia can be challenging. Relative to congenital type, presentation of acquired hemophilia is quite distinct wherein the common manifestation is bleeding into deep soft tissue

Table 2. Patient's mixing test results

\begin{tabular}{lcc}
\hline \multicolumn{1}{c}{ Blood investigation } & Immediate mixing & 2 hours incubation at $37^{\circ} \mathrm{C}$ \\
\hline Patient neat PT (s) & - & - \\
Patient neat aPTT (s) & 36.6 & 38.2 \\
Patient mix PT (s) & 14.0 & - \\
Patient mix aPTT (s) & 63.5 & 62.0 \\
Control normal PT (s) & - & - \\
Control normal aPTT (s) & 44.1 & 38.2 \\
\hline
\end{tabular}

Interpretation: possible presence of inhibitor.

PT, prothrombin time; aPTT, activated partial thromboplastin time. 
(hematoma) or purpura, and it rarely involves joints. ${ }^{3)}$ In our case, the sudden presence of spontaneous spontanous hematoma in an elderly patient without a history of bleeding disorder or trauma should have raised high clinical suspicion for acquired hemophilia. However, it was uncorrected aPTT from plasma mixing test that confirmed the diagnosis of acquired hemophilia. Excluding lupus anticoagulant test results, reduced factor VIII levels and the presence of factor VIII inhibitor were critical for confirming the diagnosis of acquired hemophilia A (Figure 1). ${ }^{3,6)}$

The disease is potentially life-threatening with mortality in the range of $8 \%-22 \%$, and the patients are at highest risk in the first few weeks following the initial clinical presentation. Mortality remains high among the elderly population, with a rate of $50 \%$ at 1 year following diagnosis. $^{2,7)}$

Treatment of acquired hemophilia includes immediate hemostasis and immunosuppression, and this helps in controlling the autoantibody production. The usual treatment involves administration of a high dose of oral prednisolone at $1 \mathrm{mg} / \mathrm{kg}$ combined with oral cyclophosphamide 50-100 mg per day for up to 2 months with regular aPTT monitoring. ${ }^{3)}$ Most patients respond well to such combination treatment regime, and monitoring of aPTT is sufficient to study the effectiveness of the treatment in patients. It has recently been shown that treatment of acquired hemophilia with rituximab is more effective and well tolerated. It has also been found to be effective in cases where the conventional treatment is either not responsive or recommended. ${ }^{4)}$ Treatment with rituximab involves the intravenous infusion of 375 $\mathrm{mg} / \mathrm{m}^{2}$ in each administration at weekly interval for a total of 4 weeks. Treating patients with this drug has shown promising results, with an increase in plasma factor VIII levels and a corresponding fall in inhibitor titer within the first week of treatment.

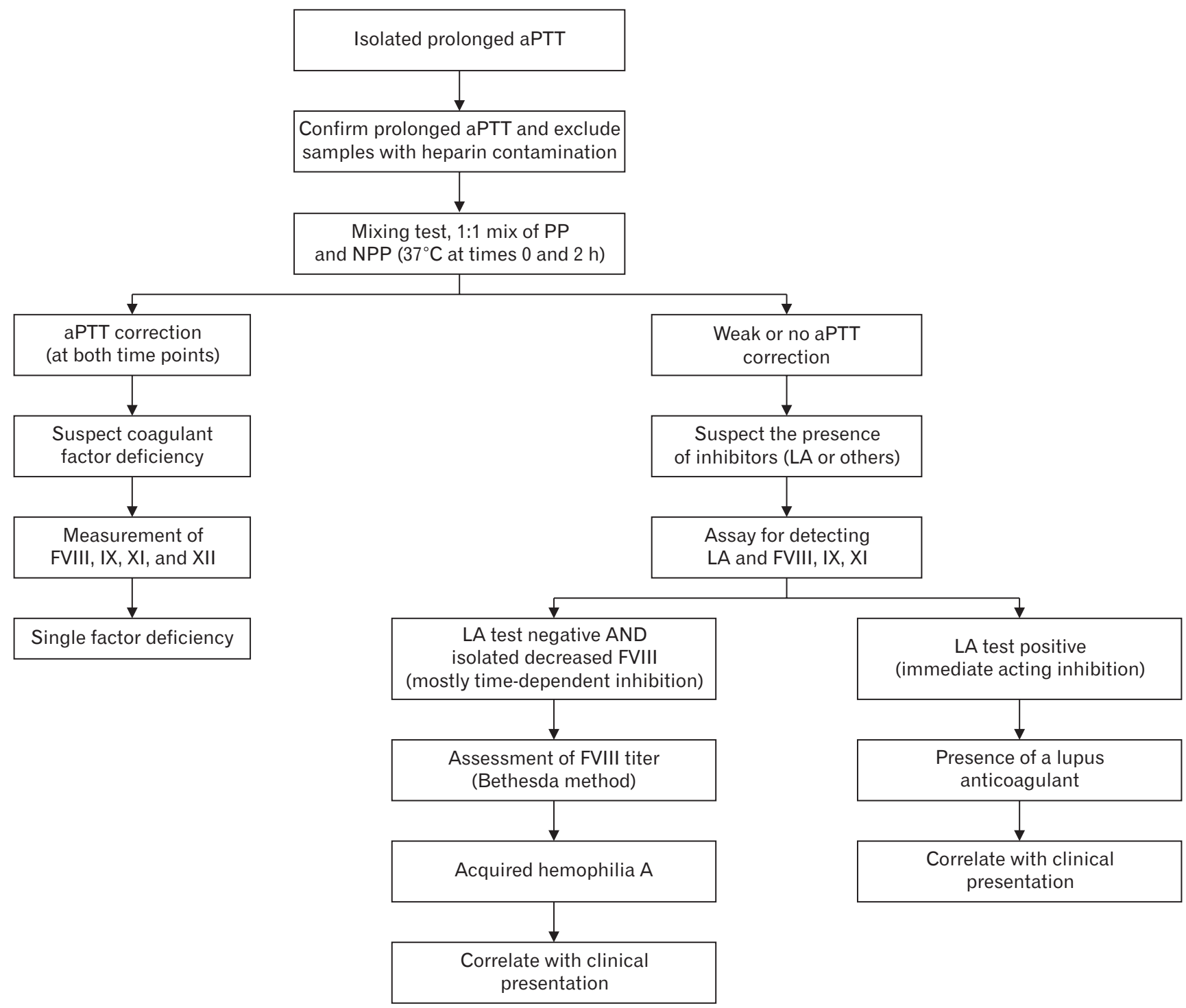

Figure 1. A diagnostic algorithm for the laboratory diagnosis of acquired hemophilia A. From Mulliez et al. Int J Lab Hematol 2014;36:398-407.6) aPTT, activated partial thromboplastin time; PP, patient plasma; NPP, normal pooled plasma; LA, lupus anticoagulant; F, coagulant factor. 
From the current study, it is evident that at initial presentation, diagnosis of acquired hemophilia is not given a priority; further, the isolated prolonged aPTT finding is often ignored. It is critical for the primary health care provider to perform a complete assessment and be aware of rare yet clinically relevant diseases. Considerable attention should be paid while examining the patient, and appropriate investigations should be carried out without any delay. Implications of under-recognized or delayed diagnosis of such diseases can be disastrous to the patient. Early treatment of diseases, such as acquired hemophilia, can significantly reduce the mortality and morbidity resulting from such conditions, particularly for those effective treatments is available.

\section{CONFLICT OF INTEREST}

No potential conflict of interest relevant to this article was reported.

\section{REFERENCES}

1. Coutre S, Leung LL, Tirnauer JS. Acquired inhibitors of coagulation [Internet]. Riverwoods (IL): UpToDate; 2017 [cited 2017 Nov 1]. Avail- able from: http://www.uptodate.com/contents/acquired-inhibitorsof-coagulation.

2. Grethlein SJ, Kessler CM, Nagalla S. Acquired hemophilia [Internet]. New York (NY): Medscape; 2016 [cited 2017 Nov 1]. Available from: http://emedicine.medscape.com/article/211186-overview.

3. Kessler CM, Knobl P. Acquired haemophilia: an overview for clinical practice. Eur J Haematol 2015;95 Suppl 81:36-44.

4. Giangrande P. Acquired hemophilia [Internet]. Montreal (Que): World Federation of Hemophilia; 2012 [cited 2017 Nov 1]. Available from: http://www1.wfh.org/publications/files/pdf-1186.pdf.

5. Collins PW, Hirsch S, Baglin TP, Dolan G, Hanley J, Makris M, et al. Acquired hemophilia A in the United Kingdom: a 2-year national surveillance study by the United Kingdom Haemophilia Centre Doctors' Organisation. Blood 2007;109:1870-7.

6. Mulliez SM, Vantilborgh A, Devreese KM. Acquired hemophilia: a case report and review of the literature. Int J Lab Hematol 2014;36:398407.

7. Girault S, Ly K, Jaccard A, Loustaud V, Turlure P, Julia A, et al. Prognosis of acquired hemophilia in older people. J Am Geriatr Soc 2008;56: 956-8. 